\title{
Hallazgo de un acueducto precolombino en la cuenca media del río Pacuare, Costa Rica
}

\author{
Ana Cristina Hernández Alpízar*
}

\begin{abstract}
RESUMEN
En el marco de la actualización del Estudio de Impacto Ambiental (EsIA) del Proyecto Hidroeléctrico Pacuare elaborado por el Instituto Costarricence de Electricidad (ICE) se detectó, mediante una prospección arqueológica intensiva, evidencia precolombina de suma importancia en el ámbito nacional, como lo fue el hallazgo del segundo asentamiento con obras hidraúlicas conocido hasta el momento en el país. El artículo es la adaptación de un segmento del apartado sobre la arqueología del PH Pacuare (Hernández y Ovares, 2005): Memoria Proyecto de desarrollo Hidroeléctrico Pacuare. ICE, 2005.

Palabras clave: Arqueología, Sitios arqueológicos, Acueductos, Río Pacuare (Costa Rica)

ABSTRACT

The activities necessary for the completion of the Environmental Impact Assessment for the Pacuare Hydroelectric Project revealed, through intensive archaeological surveys, extremely important Pre-Columbian evidence at the national level the find of the second settlement known in the country with hydraulic structures. The article is an adaptation from a segment in the archaeological section of the PH Pacuare (Hernández, A.C. and E., Ovares, 2005): Memoria Proyecto de Desarrollo Hidroeléctrico Pacuare, ICE, 2005.
\end{abstract}

Keywords: Archaeology, Archaelogical Sites, Aqueducts, Pacuare River (Costa Rica)

\section{Introducción}

Las investigaciones arqueológicas realizadas por el ICE en diferentes zonas del país se remontan al año 1980 formando parte de los estudios ambientales para obras de desarrollo eléctrico, en la mayoría de los casos vía consultorías. A partir del año 2000 la labor arqueológica se intensifica y se le da la importancia que merece dentro de un equipo multidisciplinario que elabora Estudios de Impacto Ambiental, creando así estrategias para la protección del Patrimonio Arqueológico de

\footnotetext{
* Ana Cristina Hernández Alpízar. Costarricense. Licenciada en Arqueología. C.S. Gestión Ambiental, Instituto Costarricense de Electricidad, AHernandez@ice.go.cr
} 
acuerdo al marco legal vigente en Costa Rica. En la actualidad se tienen contratados arqueólogos en la mayoría de los proyectos en construcción, generando cantidad de información relevante para reconstruir la historia antigua de zonas casi inexploradas arqueológicamente, constituyendo un aporte significativo al conocimiento arqueológico del país.

A finales del año 2004 y principios del 2005 se realizó una prospección arqueológica superficial intensiva en el área de influencia del Proyecto Hidroeléctrico Pacuare (PHP), ubicado en la cuenca media del Río Pacuare, Vertiente Caribe de Costa Rica.

Como resultado de la prospección citada se reportó la presencia de 7 sitios precolombinos, además de los 7 localizados en estudios anteriores. En su mayoría se ubican en las terrazas no inundables a ambas márgenes del río Pacuare, con preferencia en la confluencia de ríos secundarios o quebradas de mayor tamaño. Así mismo se logró identificar 9 yacimientos más en el área de influencia del Proyecto que incluyó los pueblos de Pacayitas, El Progreso, San Joaquín y Surtubal (Centro Científico Tropical, 1995).

Un aspecto relevante de las investigaciones realizadas en el área del PH Pacuare es la presencia de un sitio precolombino denominado El Escobal (C-44 EE). Este es un asentamiento multicomponente, o sea presenta diferentes momentos de ocupación en un rango temporal de 300 años antes de Cristo al 1350 después de Cristo; se caracteriza por su complejidad arquitectónica ya que posee montículos (circulares, ovalados), basamentos, muro de contención, camino empedrado (calzada), áreas funerarias, rampa o escalinatas de acceso a las estructuras.

Probablemente fue habitado por personajes importantes a nivel local que controlaban o administraban este territorio, esto lo constituye en un centro político y económico de importancia en la cuenca media del río Pacuare.

Como parte de la investigación señalada anteriormente se visitó el sitio arqueológico Rosa María situado geográficamente en coordenadas Lambert Este 582132.50 y Norte 207420.13 de la hoja cartográfica Tucurrique escala 1:50 000 del Instituto Geográfico Nacional, a una altura de 910 metros sobre el nivel del mar. Rosa María esta emplazado en una terraza elevada de la margen derecha del río Pacayitas y abarca dos niveles más de terrazas seguidas por la fila montañosa. Este yacimiento precolombino al igual que El Escobal, es de gran importancia en la zona y se localizó en Pacayitas (área de influencia del proyecto), el sitio presenta áreas funerarias, habitacionales, petroglifos y un acueducto. A pesar de que la mayoría de los rasgos se encontraron parcialmente alterados, aún permanecen estructuras que pueden generar información muy valiosa como es el caso de obras hidráulicas, ya que en el país solo se conoce una y corresponde al sitio arqueológico Guayabo de Turrialba (Figura 1).

El sistema hidraúlico reportado en Guayabo de Turrialba declarado Monumento Nacional mantiene en la actualidad su funcionamiento original, pues recolecta las aguas de las nacientes situadas en la parte baja de la colina de los chamanes (situada al noroeste del complejo arquitectónico) mediante una toma principal, luego la encauza por un canal de conducción hasta el tanque disipador, de este pasa al tanque de sedimentación y luego al puente y canal de desfogue. El sistema garantizó que en el tanque de sedimentación se pudiese recolectar el agua 
(potable) de muy buena calidad que posiblemente fue utilizada en tiempos precolombinos para el consumo humano, recreativo, culinario y elaboración de artesanía. Los indígenas que diseñaron y construyeron Guayabo aprovecharon la topografía del terreno para la utilización de los recursos hidraúlicos, así como para ubicar las diferentes obras de infraestructura (Aguilar, 1972; Fonseca, 1978; Dubón y Solís, 1981; Hurtado de Mendoza, 2004).

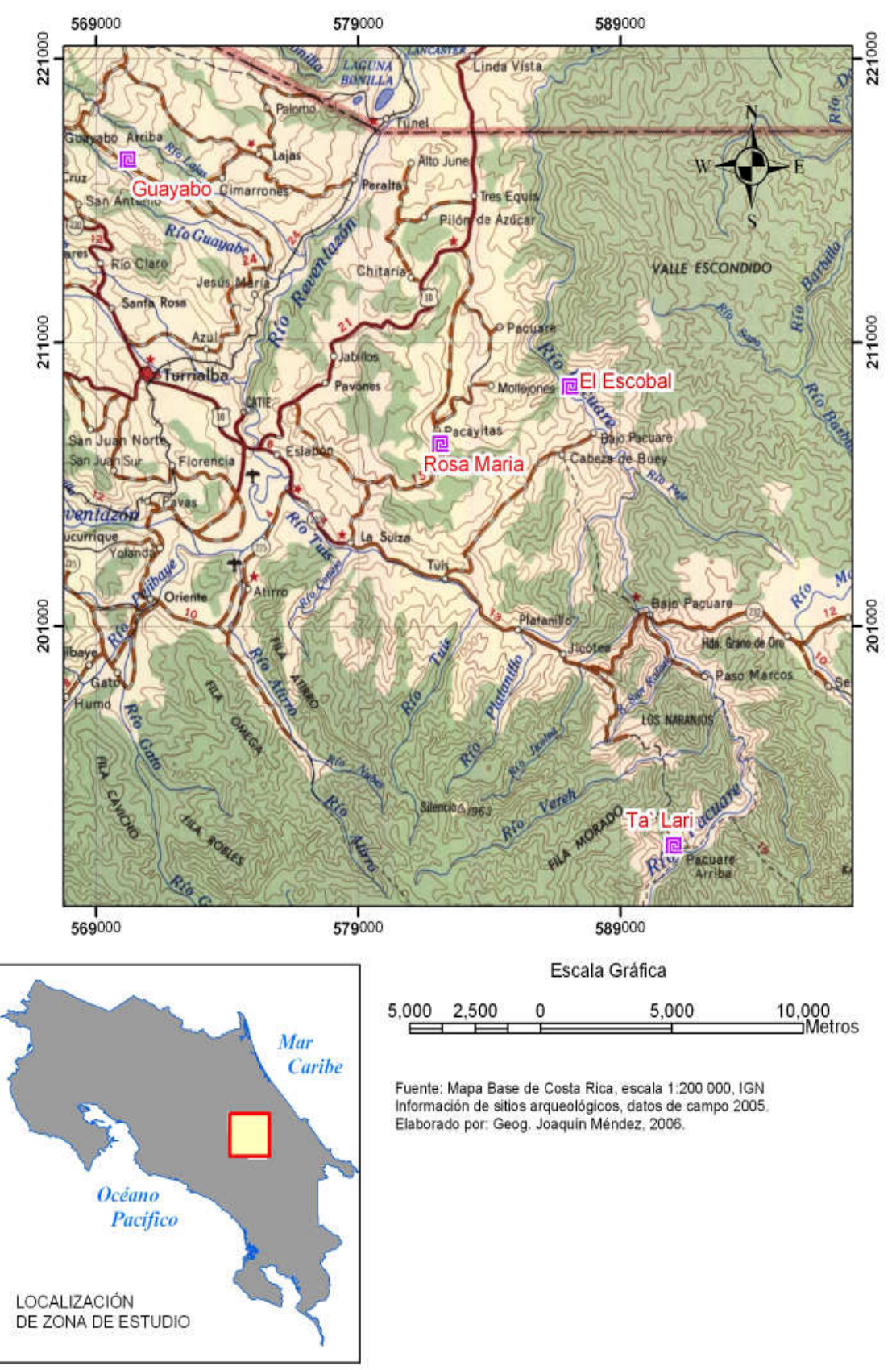

Figura 1: Ubicación de sitios arqueológicos 


\section{Antecedentes}

El sitio Rosa María fue reportado por el arqueólogo norteamericano William Kennedy en 1968, en dicha oportunidad registró dos rasgos arquitectónicos. El primero de ellos se ubicó a $40 \mathrm{~m}$ al oeste de la casa de los antiguos propietarios de apellido Núñez, según el autor en primera instancia interpretó este rasgo como un basamento $(13.7 \mathrm{~m}$ de diámetro) de una vivienda indígena y posteriormente sugiere su carácter funerario. En este rasgo efectuó dos pruebas

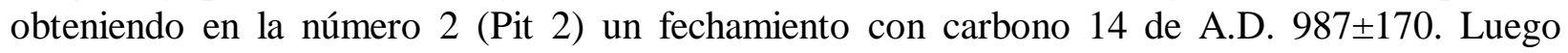
menciona el Rasgo 2 que se ubica al sur del anterior (R-1) y que presenta pequeños cúmulos de tierra de baja altura con piedras que sobresalen a lo largo de sus lados, desconociendo su naturaleza (Figura 2).

\section{Figura 2}

\section{Croquis del sitio arqueológico Rosa María}

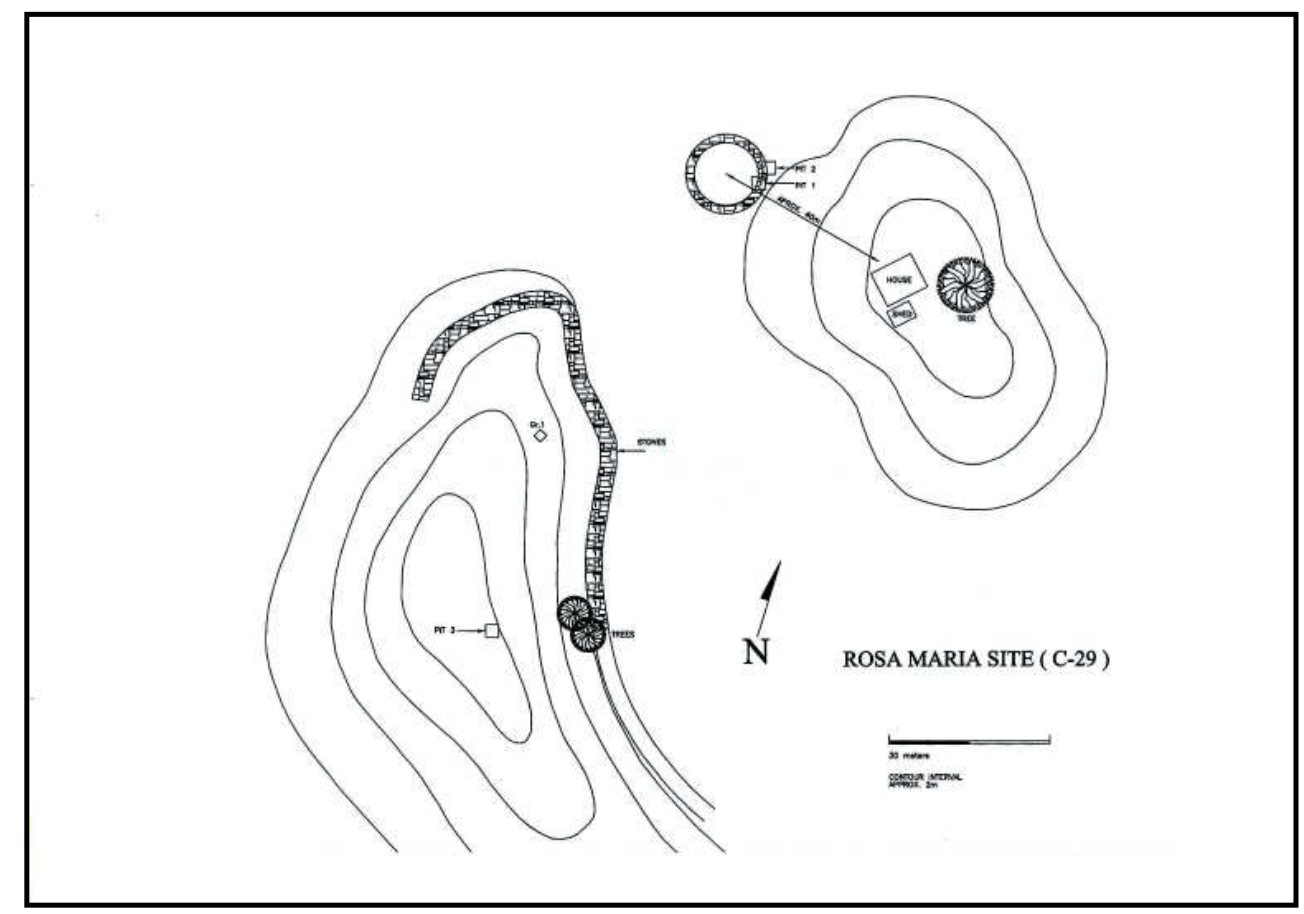

Fuente Kennedy, 1968

Kennedy también excavó una tumba en un sector funerario del sitio Rosa María (26 m al suroeste del Rasgo 1 en la cima de una colina), el rasgo funerario se encontró a $21 \mathrm{~cm}$ de profundidad, tenía paredes de cantos rodados, tapa de lajas y el piso carecía de piedras, se orientaba de Norte-Sur (medía $1.81 \mathrm{~m}$ x $1.25 \mathrm{~m}$ ). En este enterramiento se halló un fragmento de femur y tres artefactos cerámicos.

Otro dato interesante en el sitio Rosa María es la presencia de cuatro grabados en piedra. El petroglifo 29 localizado a $1 \mathrm{~km}$ al norte del sitio (cerca de la escuela de Pacayitas), consiste en una espiral; el petroglifo 30 se encuentra actualmente en el Museo Nacional de Costa Rica y 
originalmente se reportó cerca del rasgo 1 también presentaba una espiral. Por último se describe los petroglifos 31 y 32 a corta distancia al oeste del sitio Rosa María observándose en ellos diseños abstractos que consisten en líneas onduladas.

\section{Estudios arqueológicos recientes en el sitio Rosa María}

En la actualidad el terreno donde se encuentra el sitio Rosa María ha sufrido alteraciones propias de las actividades agrícolas, anteriormente el cultivo del café, la presencia de un trapiche y ahora la caña de azúcar, así como la construcción de nuevas viviendas. Ejemplo de ello según narró uno de los propietarios del terreno que al cavar la tierra para fundar los cimientos de su casa encontraron algunas tumbas de cajón con paredes de cantos rodados y grandes tapas de lajas similares a las descritas por Kennedy, en ellas aparecieron artefactos cerámicos y líticos. Otros vecinos mencionaron que al abrir hoyos para basura u otras labores de la finca se hallaron diferentes artefactos en piedra y cerámica (Figura 3).

\section{Figura 3}

\section{Piezas cerámicas y líticas halladas durante la construcción de una vivienda}

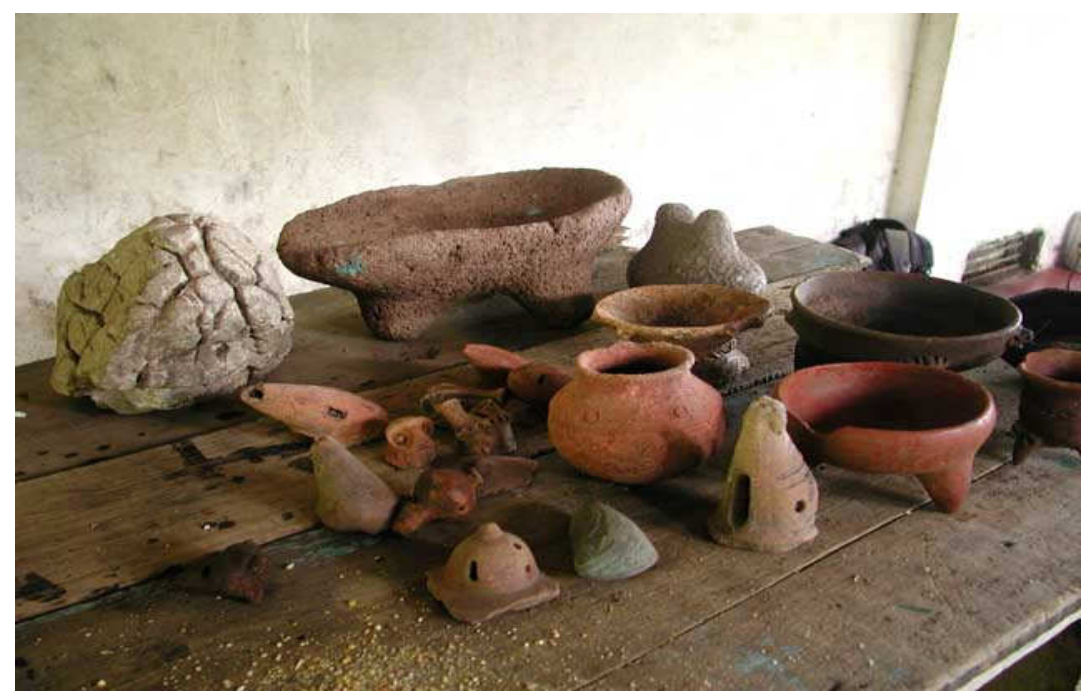

En un nivel superior de la terraza donde se encuentra la casa del dueño actual Sr. Vianney Araya y parte de las estructuras precolombinas, existen dos terrazas de menor tamaño con presencia de evidencia arqueológica. Al iniciarse la zafra de la caña se logró explorar el lugar, reportándose algunos rasgos funerarios alterados por saqueadores, maquinaria y por los cultivos. Según el propietario de la finca en la tercera terraza se pueden localizar cantidad de piedras dispersas y entre ellas fragmentos de cerámica precolombina, es posible que correspondan a rasgos habitacionales y funerarios muy perturbados. También se detectó filas de piedras o muros en sentido transversal a lo largo de las terrazas muy afectados por el paso de maquinaria, es posible que alguno de estos muros coincida con el rasgo 2 descrito por Kennedy. Además, en este sector se hallaron dos petroglifos más cuyo diseño consiste en un conjunto de espirales (Figuras 4,5 y $6)$. 


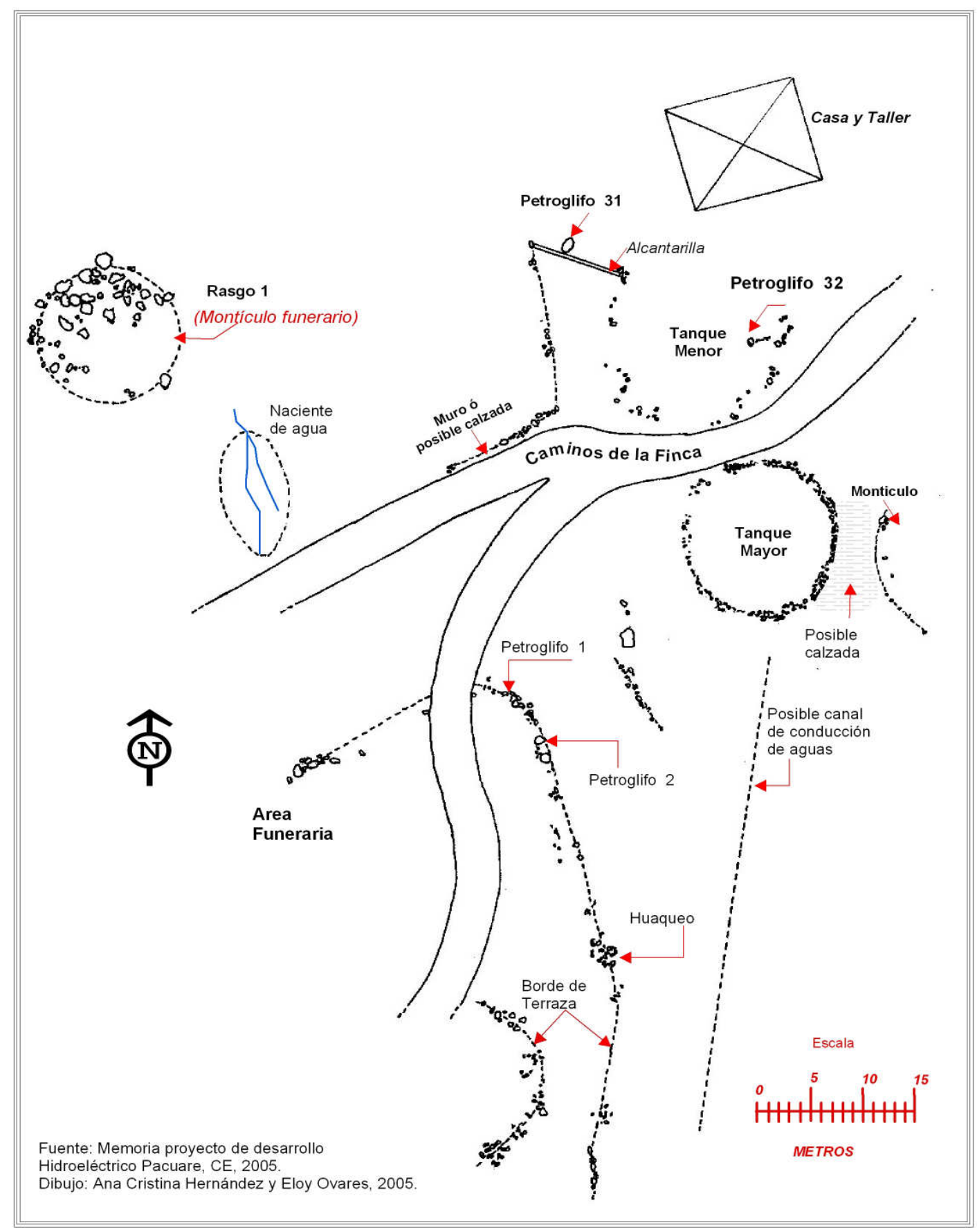

Figura 4: Sitio arqueológico Rosa María 

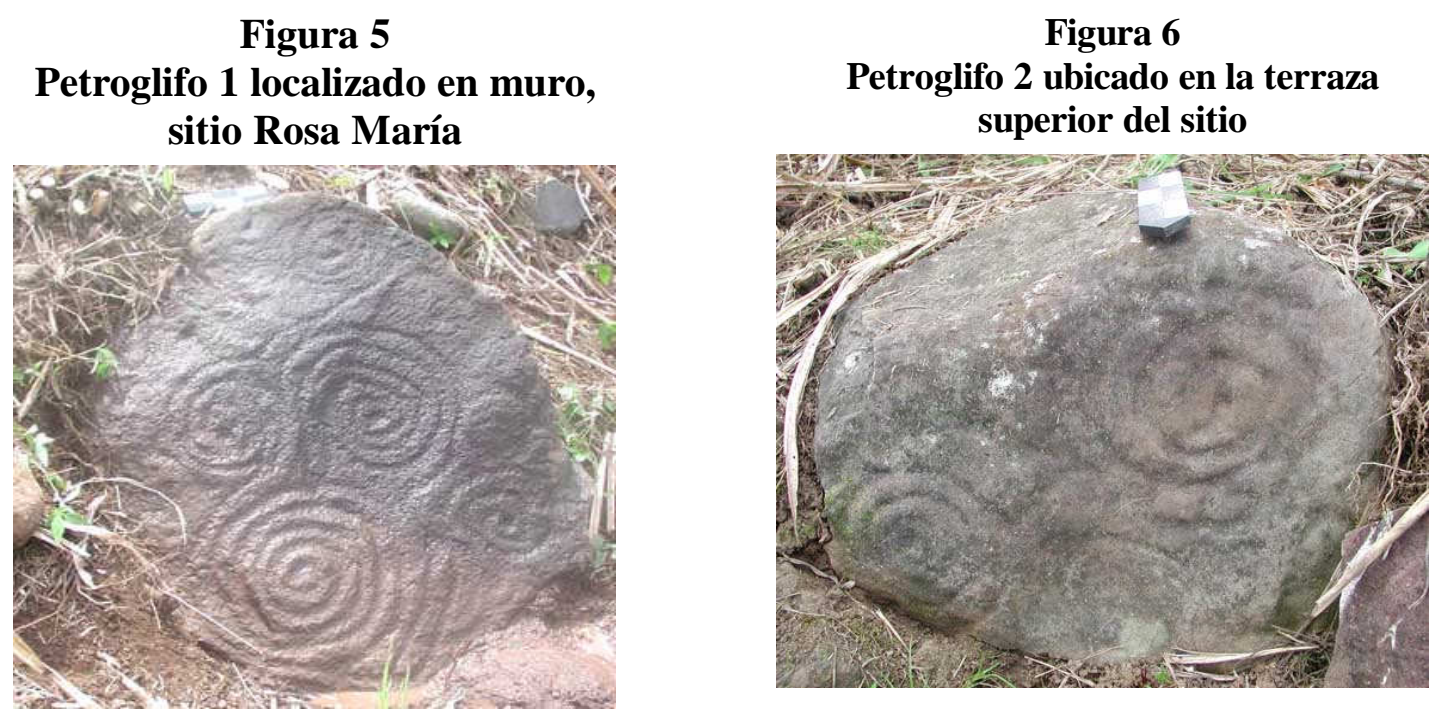

El rasgo 1 reportado por Kennedy se encuentra totalmente perturbado, observándose cantos de gran tamaño dispersos por toda el área, esto se debe quizás a que se encuentra a escasos metros del camino principal y muy cerca de una chanchera situada en la margen derecha del río Pacayitas.

\section{Hallazgo de un sistema de obras hidráulicas}

A pesar de la alteración que ha sufrido el sitio Rosa María, se logró detectar a escasos metros de la casa del dueño, la existencia de parte de un sistema de obras hidraúlicas semejantes a las de un acueducto. El primer rasgo consiste en una estructura circular (denominado tanque mayor) de aproximadamente $12.5 \mathrm{~m}$ de diámetro, su anillo (pared) esta construido con cantos rodados. En un segmento de la pared al lado este aún se conserva parte de la estructura original que posee $1.06 \mathrm{~m}$ de altura. En el resto del muro fueron removidas las alineaciones superiores, desplazándolas al camino adyacente de la finca, en los alrededores de la estructura y posiblemente dentro de la misma, ya que al sondear el interior del círculo se detectó piedra a diferentes profundidades (entre 0.40 a $1.20 \mathrm{~m}$ ) (Figura 7 ).

\section{Figura 7}

\section{Sistema hidraúlico, tanque mayor del sitio Rosa María}

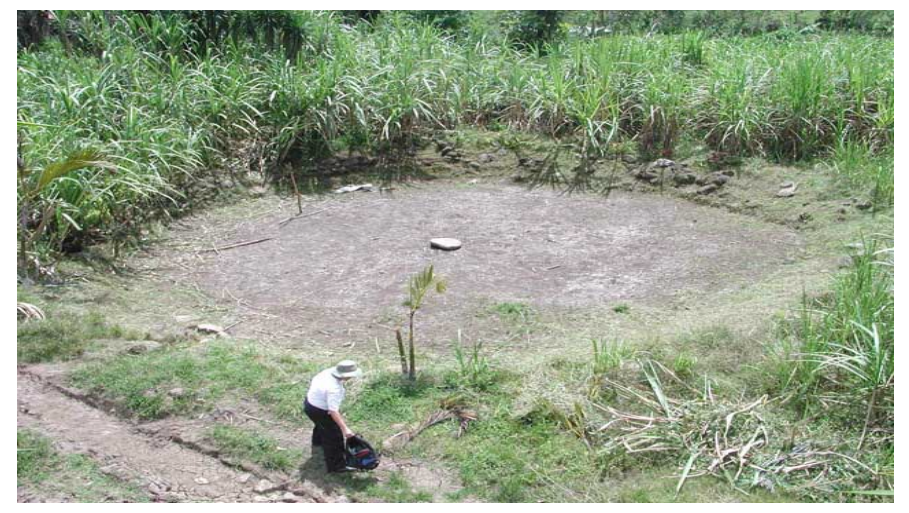


También se planteó como hipótesis que la presencia de rocas dentro de la estructura se debe a la existencia de un piso, sin embargo, solo realizando una excavación se obtendrán mayores datos al respecto.

Según el Sr. Araya las aguas pluviales se depositan en la estructura mencionada anteriormente y luego es absorbida en su interior, narra que pretendía aprovechar esta estructura para realizar un criadero de tilapia. Para esto excavó dos hoyos cerca de la pared norte y los llenó de agua pero esta no permanecía allí, sucedió que se formaba una especie de remolino y era absorbida, por ello ideó forrar el suelo con plástico negro pero tampoco lo consiguió, aún se puede observar parte del mismo en superficie.

En el sector noroeste del rasgo citado se localiza el camino de la finca (con un ancho de $4 \mathrm{~m}$ ) inmediatamente después existe otra estructura denominada tanque menor cuya forma es difícil de determinar por la cantidad de maleza, rocas y tierra que se encuentra sobre ella, podría ser circular u ovalada con un diámetro aproximado de $10 \mathrm{~m}$ y $0.95 \mathrm{~m}$ de altura. Con la construcción del camino es posible que se halla alterado parte del acueducto pues precisamente en este lugar se observó una especie de canal de conducción ( $\mathrm{S}-\mathrm{N})$ que dirigía las aguas del primer rasgo al segundo, lo cual explicaría por qué las aguas no permanecen en la primera estructura.

En el segundo rasgo se encontró un petroglifo muy erosionado, su diseño está compuesto por una espiral y algunas líneas onduladas, es posible que lo hallan trasladado y reutilizado en la construcción de la estructura. En el sector noroeste del tanque menor se halló un orificio de forma rectangular $(0.20 \mathrm{~m}$ alto $\mathrm{x} 0.30 \mathrm{~m}$ ancho) conformado por dos hileras de rocas (paredes laterales) cuyo tamaño promedio es de $0.23 \mathrm{~m}$ y sobre ellas un revestimiento de rocas de mayor tamaño $(0.60 \mathrm{~m})$ colocadas horizontalmente $(0.30 \mathrm{~m}$ de la superficie). Aguas abajo del orificio existe una especie de alcantarilla de $10.5 \mathrm{~m}$ de longitud que podría haber funcionado como canal de desfogue y se comprobó que aún funciona (Figura 8).

\section{Figura 8}

\section{Orificio de forma rectangular donde se introduce el agua hacia una especie de alcantarilla}

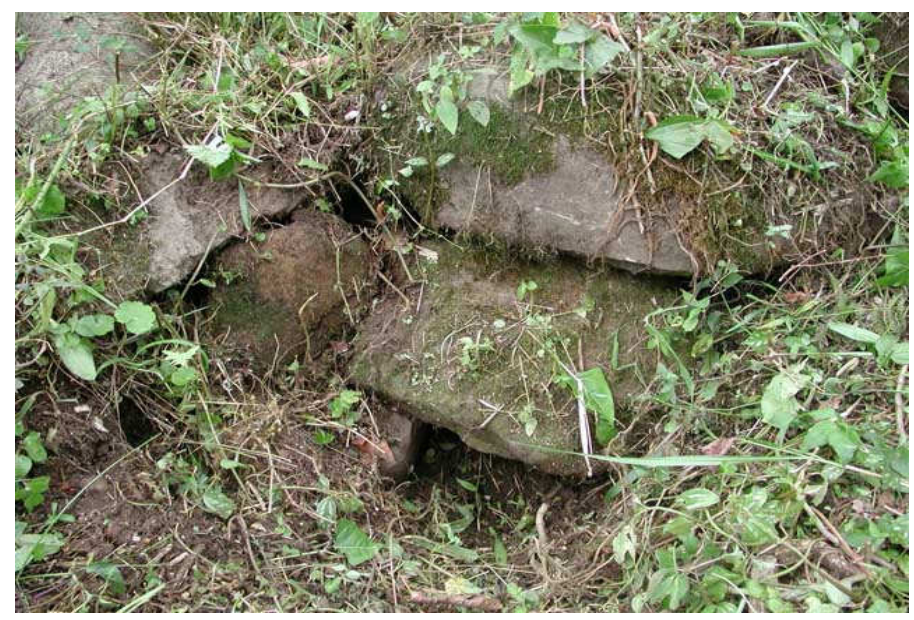


El canal de desfogue referido en el párrafo anterior restituye las aguas al río Pacayitas por otro orificio con las mismas características que el anterior, variando un poco en sus dimensiones $(0.26$ $\mathrm{m}$ de alto $\mathrm{x} 0.28 \mathrm{~m}$ de ancho) y encontrándose a $1.30 \mathrm{~m}$ con respecto a la superficie y es posible que sobre él exista un puente (cantos o lajas). Esto se deduce por la existencia del petroglifo 31 (elaborado en alto relieve, cuyo diseño es abstracto posiblemente la estilización de un animal) cerca del lugar y la presencia de un muro de piedra que podría tratarse de una calzada, pero fue barrida por maquinaria para ampliar el camino de la finca. Actualmente solo se puede observar parte del muro ( $0.60 \mathrm{~m}$ de altura) en el perfil del camino ya que existe una pendiente pronunciada hacia el río Pacayitas. Este muro según narró el Sr. Araya se prolonga alrededor de la vivienda siguiendo el contorno del terreno, por lo tanto, se sondeó comprobando su continuidad en algunos sectores, sin embargo, es necesario realizar unas pruebas para constatarlo (Figuras 9 y 10).

Figura 9

Orificio de salida (canal de desfogue) que restituye las aguas al río Pacayitas

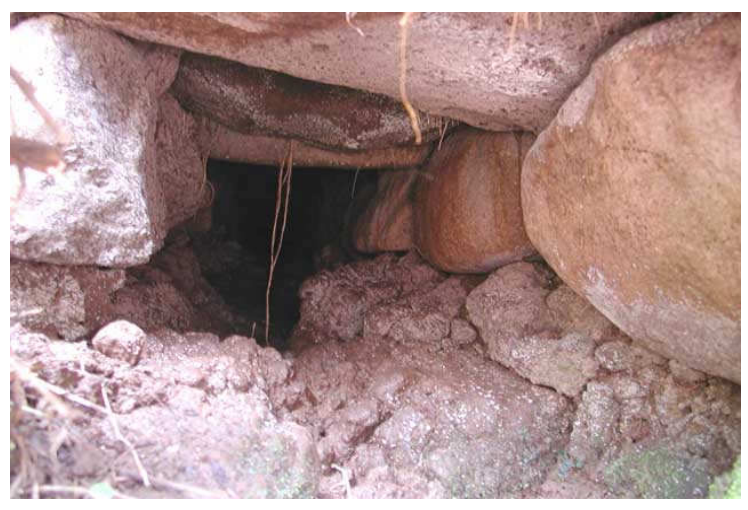

Figura 10

Petrogilfo 31, diseño abstracto

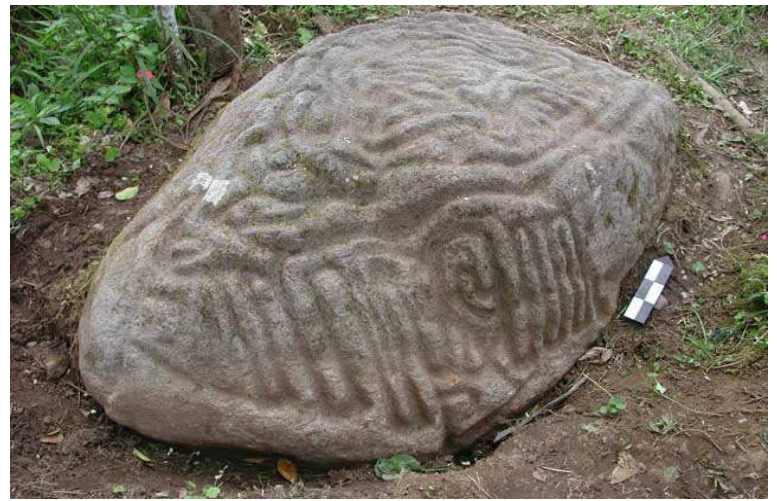

El acueducto no funciona en su totalidad ya que la toma principal situada probablemente en el sector de mayor altura del terreno ha sido removido quizás obstruyendo el canal de conducción hacia la primera estructura que tal vez funcionaba como un tanque de sedimentación para purificar el agua que luego se dirigia al tanque menor.

Aprovechando la época de safra de la caña en esta área, se invitó al Ing. Ricardo Baldi Alvarado, del Área Ingeniería Hidráulica-CSD-ICE, a visitar el sitio para analizar el acueducto. Según Baldi el sector suroeste presenta las características topográficas necesarias para transportar agua por gravedad desde alguna toma de aguas situada en la zona alta hacia las estructuras de almacenamiento en la parte baja. A raíz de esta hipótesis se sondeó el sector noroeste de la primera estructura (tanque mayor) obteniendo como resultado una alineación de cantos en esa direccción que, sin dejar de lado el estado de pertubación del lugar, insinúan la existencia de un canal de conducción.

Cercano al sitio existen tanto aguas superficiales (río Pacayitas y quebrada afluente) como subterráneas (al menos una naciente). Lo más probable es que el agua se captó de alguna naciente ya que el terreno hacia el oeste presenta mayor elevación y las aguas superficiales 
cercanas se encuentran a un nivel inferior. Hoy en día no se evidencia una naciente en la zona alta pero existe la posibilidad de que tanto la deforestación como el cambio del uso del suelo hallan alterado las características hidrogeológicas del sitio y hallan secado dicha naciente. Otras hipótesis propuestas por Baldi son:

- Quizás el tanque mayor se usó como una especie de "embalse de regulación horaria" durante la estación seca precisamente para almacenar el agua de la naciente ya que en la actualidad mientras las quebradas cercanas se secan durante esta estación la naciente arriba citada muestra una producción baja pero continua.

- Quizás el tanque mayor abastecía a la población y al crecer ésta fue necesario ampliar la capacidad de almacenamiento mediante la construcción de un segundo tanque.

A unos $3 \mathrm{~m}$ al este del tanque mayor se descubrió parte de un montículo aparentemente de forma circular y de grandes dimensiones construido con cantos rodados, se encontró sumamente alterado ya que sobre el existen algunas casas e incluso la presencia de un viejo trapiche y cultivos. En una de las casas mencionadas propiedad del Sr. Nájera se encontró en su jardín el petroglifo (3) que consta de una espiral y por sus dimensiones $(0.40 \mathrm{~m}$ de diámetro y con un grosor de $0.20 \mathrm{~m}$ ) se infiere fue traslado hasta este lugar. Al sondear el espacio entre el tanque mayor y el montículo se detectó la presencia de cantos bajo superficie, es posible que se trate de una calzada (Figura 11).

\section{Figura 11 \\ La flecha señala el sector de montículo muy alterado en la parte superior}

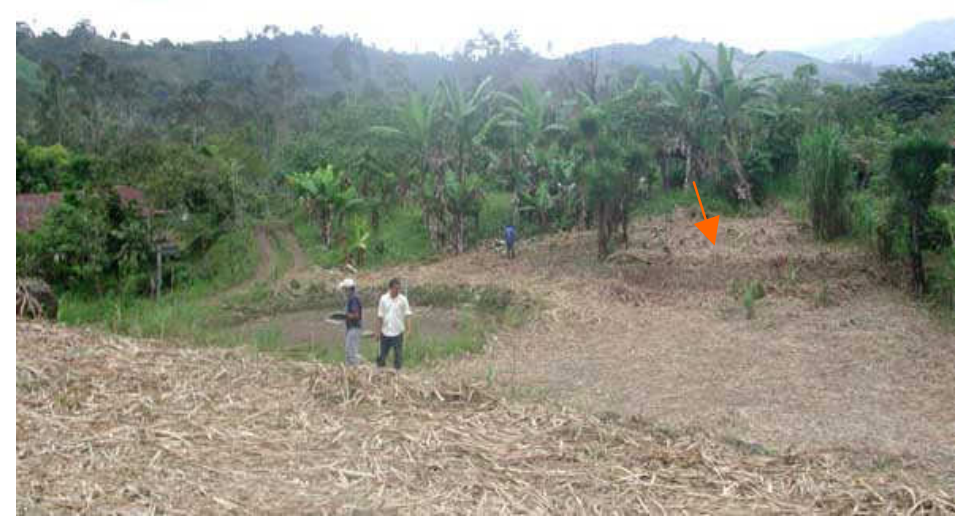

Un detalle interesante del sitio Rosa María es que se ubica cerca de donde nace el río Pacayitas, por lo tanto si se navegara por el río aguas abajo, a unos 5 ó $6 \mathrm{~km}$ se llegaría a su desembocadura en el río Pacuare, precisamente donde se encuentra El Escobal. Tomando en cuenta la temporalidad y complejidad de ambos asentamientos arqueológicos es probable el contacto entre ellos vía fluvial. De igual manera con otros yacimientos ubicados en las márgenes del río Pacuare aguas arriba como lo fue el sitio monumental Ta' Lari (UCR-282) reportado por la Universidad de Costa Rica en Pacuare Arriba y aguas abajo hasta llegar a la línea costera. 


\section{Conclusiones}

Hasta el momento se tiene conocimiento de tres sitios arqueológicos de gran complejidad e importancia en la cuenca media del río Pacuare, ellos son Rosa María, El Escobal y Ta' Lari. Es posible que estos sitios dominaran o controlaran grandes extensiones de terreno, no solo en la cuenca media del río Pacuare sino también en áreas adyacentes y es probable que no sean los únicos, ya que algunos lugareños mencionan la existencia de otros sitios con montículos, calzadas, cementerios, en lugares tales como Tayutic, Vereh y Roca Blanca. Si se corrobora su existencia en futuras investigaciones se podrían aclarar algunas interrogantes en el sentido de las posibles relaciones políticas, económicas y religiosas que pudieron haberse mantenido entre ellos.

El dominio que alcanzaron estas poblaciones indigenas en cuanto al diseño y construcción de los yacimientos arqueológicos requirió de un planeamiento y conocimiento del entorno en manos de especialistas; donde se requirió mayor cantidad de mano de obra y es clara la centralización del poder en estos lugares, pues en sus alrededores se reportaron sitios menores donde fue posible determinar la existencia de cementerios y áreas habitacionales quizás construidos de materiales perecederos como lo fue la madera. Dentro de ese engranaje se halla el manejo de los recursos hídricos reportados tanto en Guayabo de Turrialba como en Rosa María para satisfacer necesidades propias del grupo como lo fueron calidad y disponibilidad, fácil acceso, controlar la saturación del agua en las estructuras, usos culinarios y domésticos, mágico-religiosos, etc.

La obtención de un inventario de los recursos arqueológicos en los estudios ambientales de proyectos de desarrollo (eléctrico, industrial, urbanístico, etc) es primordial, ya que son el punto de partida para generar estrategias de gestión para la protección del patrimonio arqueológico. En este caso el hallazgo del sistema de obras hidraúlicas en el asentamiento Rosa María requiere una investigación exhaustiva para comprobar las hipótesis planteadas y conocer con mayor detalle su función; así como la documentación de nuevos sitios arqueológicos en el área de influencia de los proyectos, ampliando de manera significativa el conocimiento sobre los grupos indígenas a través de su desarrollo histórico en este sector Caribeño poco conocido arqueológicamente.

\section{Reconocimientos}

Mi agradecimiento a Joaquín Méndez por la elaboración de las figuras 1 y 4 que acompañan este documento. A Dora Carias que me ayudó con la traducción del resumen, a Victoria Villalobos, Gerardina Araya, Alfredo Calderón por sus comentarios y a Ricardo Baldi por su visita y aportes a la interpretación.

Al arqueólogo Eloy Ovares y trabajadores de campo Andres Umaña y Melvin Calderón. Muchas gracias al Sr. Vianny Araya, propietario del terreno, por su amabilidad al permitirnos ingresar a la propiedad y por su interés en la conservación del sitio. 


\section{Literatura consultada}

Aguilar, C.H. (1972). Guayabo de Turrialba Arqueología de un sitio indígena prehispánico. San José: Editorial Costa Rica.

Centro Científico Tropical. (1995). Estudio Ambiental Preliminar Proyecto Hidroeléctrico Pacuare del Instituto Costarricense de Electricidad.

Dubón, J.; Solís, H. (1981), Evaluación de las Obras Hidráulicas del Sitio Arqueológico Guayabo. San José: Primeras Jornadas Investigación, Vicerrectoría de Investigación de la Universidad de Costa Rica.

Fonseca, O. (1979). Informe de la primera temporada de excavación de Guayabo de Turrialba. Vínculos. Vol. 5:35-52. San José: Museo Nacional de Costa Rica.

Hurtado de Mendoza, L. (2004). Guayabo: Historia Antigua de Turrialba. San José: Litografía e imprenta LIL.

Hurtado de Mendoza, L.; Gómez, F. (1985). Breve descripción comparativa de dos regiones arqueológicas en Costa Rica: Guayabo de Turrialba y Ta' Lari de Pacuare. Vínculos Vol. 11:67100. San José: Museo Nacional de Costa Rica.

Instituto Costarricense de Electricidad. (2005). Actualización del Estudio de Impacto Ambiental. Memoria: Proyecto de Desarrollo Hidroeléctrico Pacuare.

Kennedy, W. (1968). Archaeological Investigations in the Reventazon River Drainage Area, Costa Rica. Tesis inédita de doctorado. Departament of Anthropology, Tulane University, New Orleans. 